\title{
Primordial anisotropies from cosmic strings during inflation
}

\author{
Sadra Jazayeri, ${ }^{1}$ Alireza Vafaei Sadr, ${ }^{2,3}$ and Hassan Firouzjahi ${ }^{1}$ \\ ${ }^{1}$ School of Astronomy, Institute for Research in Fundamental Sciences (IPM) \\ P. O. Box 19395-5531 Tehran, Iran \\ ${ }^{2}$ Department of Physics, Shahid Beheshti University, G.C., Evin, Tehran 19839, Iran \\ ${ }^{3}$ Département de Physique Théorique and Center for Astroparticle Physics, Université de Genève, \\ 24 Quai Ernest Ansermet, 1211 Genève 4, Switzerland
}

(Received 19 March 2017; published 13 July 2017)

\begin{abstract}
In this work, we study the imprint of an individual primordial cosmic string within a Hubble patch on the inflationary power spectrum. A straight cosmic string induces two distinct contributions to the curvature perturbations power spectrum. The first type of correction respects the translation invariance while violating isotropy. This generates quadrupolar statistical anisotropy in cosmic microwave background maps, which is constrained by the Planck data. The second contribution breaks both homogeneity and isotropy, generating a dipolar power asymmetry in the variance of temperature fluctuations with its amplitude falling on small scales. We show that the strongest constraint on the tension of primordial cosmic strings is obtained from the quadrupolar anisotropy and argue that the mass scale of the underlying theory responsible for the formation of the string cannot be much higher than the grand unified theory scale. The predictions for the diagonal and off-diagonal components of the cosmic microwave background angular power spectrum induced by the string are presented.
\end{abstract}

DOI: $10.1103 /$ PhysRevD.96.023512

\section{INTRODUCTION}

The precise measurements of anisotropies on cosmic microwave background (CMB) temperature fluctuations and its polarization maps [1-3] have provided strong support for inflation as the leading theory for early Universe and generating the initial perturbations. The basic predictions of inflation that the CMB perturbations are nearly scale invariant, nearly adiabatic, and nearly Gaussian are in good agreement with these observations.

There are indications of anomalies on CMB maps as reported in the Planck results [1,3] and also in earlier observations, such as the dipole asymmetry and the power suppressions on large scales. There are two different views as to how interpret these anomalies. One attitude is that these anomalies are not statistically significant and may be due to a lack of precise data, unknown systematics, or even methods of data analysis. This is mainly motivated by the fact that these anomalies are observed on low- $\ell$ regions of CMB maps in which the effects of cosmic variance are nonnegligible. It is possible that these anomalies are artifacts of poor statistics on large scales. In this view, no single anomaly is significant enough to challenge the simple concordance model of early Universe. It is argued that if a theoretical model can address more than one anomaly at the same time then these anomalies and the theory behind their generation become significant. The other attitude is that these anomalies may be genuine and may hint to nontrivial inflationary dynamics. If so, understanding these anomalies may open new windows to the physics of the primordial Universe. This is particularly important if the anomalies persist in current and future observations. In addition, if some theoretical models can address not only anomalies in CMB temperature maps but also provide independent predictions for $\mathrm{CMB}$ polarization maps and primordial tensor perturbations, then it is worth studying these scenarios.

In particular, the Planck data indicate the existence of a hemispherical power asymmetry in CMB maps [4,5], which was observed earlier in WMAP data, too [6-8]. Fitting the temperature anisotropy with a dipole modulation [9] in the form

$$
\Delta T(\hat{\mathbf{n}})=\overline{\Delta T(\hat{\mathbf{n}})}\left(1+A_{d} \hat{\mathbf{n}} \cdot \hat{\mathbf{p}}\right)
$$

the Planck data found the dipole amplitude $A_{d} \simeq 0.06$ with the preferred direction $\hat{\mathbf{p}}$ toward the southern hemisphere with respect to the Galactic plane. One interesting feature of the dipole asymmetry is that the amplitude of the dipole shows strong scale dependence such that it falls off rapidly on smaller scales, say for $\ell \geq 100$. The effects of dipole asymmetry in CMB data and large-scale structure have been further investigated in Refs. [10-21].

With these discussions in mind, there has been significant interest in addressing the nature of the dipole asymmetry in recent years. One interesting proposal for the mechanism of dipolar asymmetry is the idea of long mode modulations [22]. In this picture, it is assumed that there exists a mode $k_{L}$ that is much longer than the Hubble radius during inflation. This long mode generates the power asymmetry by modulating the background inflationary parameters such as the inflaton field or its velocity or by modulating the surface of the end of inflation. 
Unfortunately, this proposal does not work in simple models of inflation such as in single field scenarios. Based on the non-Gaussianity consistency condition [23], it is shown in Ref. [24], see also Ref. [25], that in models of inflation in which there is only one source for curvature perturbations the amplitude of dipole modulation is controlled by the amplitude of local-type nonGaussianity $f_{N L}$. Consequently, in these models with small (almost zero) $f_{N L}$, dipole asymmetry with large enough amplitudes cannot be generated. For this idea to work, one has to consider models beyond simple slow-roll scenarios such as the curvaton model, isocurvature perturbations, etc. For a list of various theoretical works based on long mode modulation and related ideas to generate dipole asymmetry, see Ref. [26].

Alternatively, the idea of using primordial defects during inflation to generate power asymmetry has been employed in Refs. [27,28]. In Ref. [27], it is assumed that there exists a domain wall during inflation that causes the asymmetry. It is shown that a large dipole with nontrivial scale dependence can be generated while the amplitudes of higher multipoles are suppressed as required from the Planck data. This idea was extended in Ref. [28] to the case of a primordial massive defect, such as a monopole or black hole, during inflation to generate power asymmetry. The presence of a massive defect breaks the translational invariance maximally while keeping the rotation invariance intact. The structure of power asymmetry is somewhat nontrivial as one also generates inhomogeneities in the primordial power spectra.

Another anomaly that has captured significant interest in recent years is the quadrupolar statistical anisotropy. Unlike the hemispherical (dipolar) asymmetry defined in Eq. (1), the quadrupolar statistical anisotropy represents anisotropy at individual points. Specifically, if one divides the CMB sphere in two opposite hemispheres, then both hemispheres are statistically the same while points on the same or opposite hemisphere can have different power. The quadrupolar statistical anisotropy in the curvature perturbation power spectrum $P_{\mathcal{R}}$ is usually parametrized in Fourier space $\mathbf{k}$ via $[29,30]$

$$
P_{\mathcal{R}}(\mathbf{k})=P_{\mathcal{R}}^{(0)}(\mathbf{k})\left(1+g_{*}(\hat{\mathbf{m}} \cdot \hat{\mathbf{k}})^{\mathbf{2}}\right),
$$

in which $P_{\mathcal{R}}^{(0)}(\mathbf{k})$ is the dominant isotropic power spectrum, $\hat{\mathbf{m}}$ represents the preferred (anisotropic) direction in the sky, and $g_{*}$ is the amplitude of quadrupolar anisotropy. Constraints from Planck data $[1,3,31]$ imply $\left|g_{*}\right| \lesssim 10^{-2}$.

The best known mechanism to generate quadrupolar statistical anisotropy is the scenario of anisotropic inflation based on the dynamics of a $U(1)$ gauge field during inflation; see, for example, Refs. [32-38]. In this mechanism, a background electric field is turned on during inflation so the background geometry is in the form of a
Bianchi I metric. If one couples the gauge field with the inflaton field appropriately, then one can reach the attractor regime in which the electric field energy density reaches a subleading but a constant fraction of the total energy density. This can lead to a small amount of quadrupole anisotropy.

Mathematically speaking, the quadrupolar statistical anisotropy given in Eq. (2) is defined in Fourier space, while the hemispherical asymmetry in Eq. (1) is defined in real space. To prevent confusion, we refer to the former as the statistical anisotropy, while the latter is called the power asymmetry or dipolar asymmetry.

In this work, we extend the motivation of Refs. [27,28] to the case of cosmic strings during inflation. To be specific, we consider the effects of an individual straight string in a Hubble patch during inflation. Our goal is to calculate the corrections in the curvature power spectrum and to look for the amplitude, shape, and scale dependence of the induced anisotropy and asymmetry. The imprints of primordial defects during inflation for various motivations have been studied in Refs. [39-44]. In particular, in Ref. [40], the correction to the curvature perturbation power spectrum induced by a cosmic string during inflation was obtained. In this work, we build and extend on the results of Ref. [40] to obtain the imprints of a cosmic string during inflation.

Before closing this section, we comment that, independent of the observational significance of anisotropy and asymmetry, the idea of looking for the imprints of defects during inflation is well motivated. Indeed, the formation of defects is a generic feature of symmetry breaking, which is expected to happen at various scales in the history of early Universe [45]. In particular, the idea of strings in the early Universe is interesting. In models of inflation constructed from string theory, such as in brane inflation, cosmic strings are copiously generated at the end or during inflation when the brane and antibrane in a brane-antibrane pair annihilate each other [46-51]. These are either fundamental strings (F strings) or D1 branes (D strings), which have different charges and couplings. They can combine to form junctions of $(p, q)$ strings that can have nontrivial implications for lensing and evolution of the networks of cosmic superstrings; for a review, see Ref. [52].

\section{CURVATURE PERTURBATIONS POWER SPECTRUM}

In this section, we present our setup of a cosmic string during inflation. The motivations and logic are similar to Refs. [27,28]. It is assumed that inflation is driven by a scalar field, the inflaton field $\phi$, which is slowly rolling on its nearly flat potential $V(\phi)$. Therefore, the dominant source of energy density is given by the potential $V$. The string is assumed to be a subdominant source of energy, and its effects can be treated perturbatively compared to those of the inflaton field. We consider the idealized situation in which the string's length is much larger than the Hubble 
radius during inflation so for practical purposes it is treated as a string with infinite length. We assume that all wiggles along the length of strings are wiped out so the string can be parametrized by its tension $\mu$. As usual, the relevant dimensionless parameter in the studies of cosmic string is the parameter $G \mu$, which measures the tension of the string in units of the Newton constant $G$. For our perturbative approach to be consistent and the contribution of string to energy density in a given Hubble radius $H^{-1}$ to be subdominant compared to the inflaton potential, we require that $\left(\mu H^{-1}\right) H^{3} \ll V$, which is equivalent to $G \mu \ll 1$. In addition, we work in the limit at which the thickness of string is negligible so it can be treated as a line of distribution of energy with the tension $\mu$.

If we consider the physical assumption that the string is formed because of a $U(1)$ symmetry breaking during inflation, then the thickness of the string is related to the energy scale of symmetry breaking, which is of the order $1 / \sqrt{\mu}$. Therefore, assuming the thickness of the string to be much smaller than the Hubble radius during inflation, we require $1 / \sqrt{\mu} \ll H^{-1}$, which in turn translates into $G \mu \gg$ $\left(H / M_{P}\right)^{2}$ in which $M_{P}=1 / 8 \pi G$ is the reduced Planck mass. Combining both conditions, we require $\left(H / M_{P}\right)^{2} \ll$ $G \mu \ll 1$. For typical models of inflation, we expect $H / M_{P} \lesssim 10^{-5}$ so the above condition can be easily satisfied for $G \mu \lesssim 10^{-2}$.

The upper bound on the tension of the string is $G \mu \lesssim$ $10^{-7}$ if a network of cosmic strings is assumed to generate parts of temperature anisotropies in CMB maps; for some recent works on this direction, see, for example, Refs. [53-56]. However, this bound does not apply to our case, since we do not consider a network of strings to generate perturbations on $\mathrm{CMB}$ after inflation. In our picture, we have one string in a Hubble horizon during inflation. In addition, to not complicate the thermal history of Universe after inflation, we assume that the string decays to relativistic particles during reheating so all its energy goes to radiation after inflation. In general, the latter assumption may not be necessary, so it may be relaxed if one is interested in the presence of a string after inflation.

We are interested in corrections to the curvature perturbation power spectrum induced by the string. Following the logic of Refs. [27,28], the dominant contribution in comoving curvature perturbations $\mathcal{R}$ is given by the inflaton field via

$$
\mathcal{R}=-\frac{H}{\dot{\phi}} \delta \phi
$$

in which $\delta \phi$ is the quantum fluctuation associated with the inflaton field. There are two types of contributions from the string, which, in principle, one has to take into account. First, the definition of curvature perturbation $\mathcal{R}$ in the presence of a string is modified so there will be an additional term in $\mathcal{R}$ beyond the leading term given in Eq. (3). Second, the string modifies the background geometry. As is well known, the geometry around a straight string is locally flat, while it modifies the geometry globally causing a deficit angle at the order $G \mu$ around string [57]. As argued in Refs. [27,28], the first contributions in the curvature power spectrum are at the order $G \mu \sqrt{\epsilon_{H}}$ in which $\epsilon_{H}$ is the slow-roll parameter $\epsilon_{H} \equiv-\dot{H} / H^{2}$. Intuitively speaking, the first contribution comes from the gravitational backreactions of the string on inflaton dynamics, which necessarily has both of the small parameters $G \mu$ and $\sqrt{\epsilon_{H}}$. However, the second contribution is the direct contribution of the string into background geometry, which is at the order $G \mu$ as we calculate below. Therefore, in the slow-roll limit where $\epsilon_{H} \ll 1$, the leading correction is from the second contribution, i.e., the direct contribution of the string to the geometry. This in turn induces a correction to the Hamiltonian, and its effects on the curvature perturbation power spectrum can be calculated using the perturbative in-in formalism $[23,58]$.

With these discussions in mind, now we proceed to study the effects of cosmic strings on the background geometry. As mentioned before, in a flat background, the geometry around the string is locally flat, while a deficit angle is induced around string. In an inflationary background with a near de Sitter (dS) background, one expects the above picture to hold and the string only to induce a deficit angle without changing the local geometry. Specifically, assuming the infinite string is extended along the $z$ direction, the vacuum solution of the string in a dS background in polar coordinates $(\rho, \phi, z)$ has been obtained to be [59]

$d s^{2}=-d t^{2}+a(t)^{2}\left(d \rho^{2}+(1-4 G \mu)^{2} \rho^{2} d \phi^{2}+d z^{2}\right)$,

in which $a(t)=\exp (H t)$ is the scale factor in the $\mathrm{dS}$ background. To leading order in the slow-roll correction, we have neglected the variation of $H$, which results in subleading corrections to our analysis, i.e., at the order $G \mu \sqrt{\epsilon_{H}}$ or higher. Note that the metric above is written nonperturbatively to all orders in $G \mu$. However, we are only interested in corrections to leading order in $G \mu$, so we shall expand the above metric to first order in $G \mu$. Also note that the above metric satisfies the intuition that the string does not change the local metric of spacetime and only induces a deficit angle equal to $8 \pi G \mu$.

It is more convenient to work with the Cartesian coordinate system in which the above metric is transformed into

$$
\begin{aligned}
d s^{2}= & -d t^{2}+a(t)^{2}\left(d \mathbf{x}^{2}-\frac{\epsilon}{\rho^{2}}\left(x^{2} d y^{2}\right.\right. \\
& \left.\left.+y^{2} d x^{2}-2 x y d x d y\right)\right)
\end{aligned}
$$


in which $\rho^{2}=x^{2}+y^{2}$ and we have defined the small dimensionless parameter $\epsilon$ via

$$
\epsilon=8 G \mu .
$$

We need to calculate the interaction Hamiltonian. For this purpose, we write down the action of inflaton field in the presence of a cosmic string. In our treatment, the inflaton field feels the presence of a string via a deformation of the background geometry induced by a cosmic string as given in Eq. (5). Note that in the limit in which we neglect the gravitational backreaction of the string on the inflaton field we can treat the scalar field as a nearly massless scalar field with the amplitude of quantum fluctuations $H / 2 \pi$. The rollings of the inflaton and its mass induces corrections at the order $\epsilon \sqrt{\epsilon_{H}}$ to the anisotropic power spectrum, which we neglect as mentioned before.

The action of a (nearly) massless inflaton field in the presence of the cosmic string encoded in the geometry (5) is given by

$$
S=-\frac{1}{2} \int d^{4} x \sqrt{-g} g^{\mu \nu} \partial_{\mu} \delta \phi \partial_{\nu} \delta \phi,
$$

in which $\delta \phi$ represents the quantum fluctuations of the inflaton field. Calculating the inverse metric $g^{\mu \nu}$ and the determinant $\sqrt{-g}$ to leading order in $\epsilon$, we have

$$
\sqrt{-g}=a^{3}\left(1-\frac{\epsilon}{2}\right)+\mathcal{O}\left(\epsilon^{2}\right),
$$

and

$$
\begin{aligned}
& \delta g^{x x}=\epsilon \frac{y^{2}}{a^{2} \rho^{2}}, \quad \delta g^{y y}=\epsilon \frac{x^{2}}{a^{2} \rho^{2}}, \\
& \delta g^{x y}=\delta g^{y x}=-\epsilon \frac{x y}{a^{2} \rho^{2}} .
\end{aligned}
$$

Since the interaction terms in the Lagrangian contain solely space derivatives, the Hamiltonian density $\mathcal{H}_{I}$ simply equals the Lagrangian density $-\mathcal{L}_{I}$. Plugging back the above results into the action, the leading-order interaction Hamiltonian is obtained to be

$$
H_{I}=\frac{\epsilon a(t)}{2} \int d^{3} \mathbf{x} \frac{\left(x \partial_{y} \delta \phi-y \partial_{x} \delta \phi\right)^{2}}{\left(x^{2}+y^{2}\right)} .
$$

Because we are interested in the curvature perturbation power spectrum in Fourier space, we calculate $H_{I}$ in Fourier space, yielding

$$
\begin{aligned}
H_{I}= & -\frac{a(t) \epsilon}{2(2 \pi)^{6}} \int d^{3} \mathbf{x} d^{3} \mathbf{k} d^{3} \mathbf{q} \frac{\delta \phi_{k} \delta \phi_{q}}{x^{2}+y^{2}} \\
& \times\left(y k_{x}-x k_{y}\right)\left(y q_{x}-x q_{y}\right) e^{i(\mathbf{k}+\mathbf{q}) \cdot \mathbf{x}},
\end{aligned}
$$

in which $\delta \phi_{k}$ is the amplitude of $\delta \phi$ fluctuations in Fourier space. From the above expressions for $H_{I}$, we see that the system enjoys the remnant translation and rotation symmetries around the $z$ direction, the orientation of the string.

Now, using the standard in-in formalism $[23,58]$, the corrections in two-point correlations of the inflaton field induced by a cosmic string at leading order in $\epsilon$ are obtained to be

$$
\begin{aligned}
\Delta\left\langle\delta \phi_{\mathbf{k}}\left(t_{e}\right) \delta \phi_{\mathbf{q}}\left(t_{e}\right)\right\rangle & =i \int_{0}^{t_{e}} d t^{\prime}\left\langle\left[H_{I}\left(t^{\prime}\right), \delta \phi_{\mathbf{k}} \delta \phi_{\mathbf{q}}\right]\right\rangle \\
& =-2 \operatorname{Im} \int_{0}^{t_{e}} d t^{\prime}\left\langle H_{I}\left(t^{\prime}\right) \delta \phi_{\mathbf{k}}\left(t_{e}\right) \delta \phi_{\mathbf{q}}\left(t_{e}\right)\right\rangle,
\end{aligned}
$$

in which $t_{e}$ indicates the time of the end of inflation.

Going to conformal time $d \eta=d t / a(t)$, we obtain

$$
\begin{aligned}
\Delta\left\langle\delta \phi_{\mathbf{k}}\left(t_{e}\right) \delta \phi_{\mathbf{q}}\left(t_{e}\right)\right\rangle= & 2 \epsilon(2 \pi) \delta\left(k_{z}+q_{z}\right) \\
& \times \int_{-\infty}^{\eta_{e}} d \eta^{\prime} a^{2}\left(\eta^{\prime}\right) h\left(\mathbf{k}_{\perp}, \mathbf{q}_{\perp}\right) \operatorname{Im}\left(\delta \phi_{k}\left(\eta^{\prime}\right)\right. \\
& \left.\times \delta \phi_{q}\left(\eta^{\prime}\right) \delta \phi_{k}^{*}\left(\eta_{e}\right) \delta \phi_{q}^{*}\left(\eta_{e}\right)\right)
\end{aligned}
$$

in which $\mathbf{k}_{\perp}$ represents the projection of $\mathbf{k}$ on the $x y$ plane, which is perpendicular to the orientation of the string, and we have defined the function $h\left(\mathbf{k}_{\perp}, \mathbf{q}_{\perp}\right)$ via

$$
\begin{aligned}
h\left(\mathbf{k}_{\perp}, \mathbf{q}_{\perp}\right) \equiv & \int d^{2} \mathbf{x} \frac{\exp \left(i(\mathbf{k}+\mathbf{q})_{\perp} \cdot \mathbf{x}_{\perp}\right)}{x^{2}+y^{2}} \\
& \times\left[x^{2} q_{y} k_{y}+y^{2} q_{x} k_{x}-x y\left(q_{x} k_{y}+q_{y} k_{x}\right)\right] .
\end{aligned}
$$

One can easily check that $h\left(\mathbf{k}_{\perp}, \mathbf{q}_{\perp}\right)=h\left(\mathbf{q}_{\perp}, \mathbf{k}_{\perp}\right)$. Also note that the delta function $\delta\left(k_{z}+q_{z}\right)$ in Eq. (13) is a manifestation of translation invariance along the string.

Using the form for the wave function of inflaton field

$$
\delta \phi_{k}=\frac{H}{\sqrt{2 k^{3}}}(1-i k \eta) \exp (i k \eta)
$$

and taking $\eta_{e} \rightarrow 0$, the right-hand side of Eq. (13) simplifies to

$$
\begin{aligned}
& 2 \epsilon(2 \pi) \delta\left(k_{z}+q_{z}\right) h\left(\mathbf{k}_{\perp}, \mathbf{q}_{\perp}\right) \int_{-\infty}^{0} \frac{d \eta^{\prime}}{H^{2} \eta^{\prime 2}}\left(\frac{H^{2}}{2 k^{3} q^{3}}\right)^{2} \\
& \quad \times \operatorname{Im}\left[\left(1-i k \eta^{\prime}\right)\left(1-i q \eta^{\prime}\right) e^{i(k+q) \eta^{\prime}}\right] .
\end{aligned}
$$

Now, using the relations 


$$
\operatorname{Im} \int_{-\infty(1-i \varepsilon)}^{0} d \eta^{\prime} e^{i(k+q) \eta^{\prime}}=\frac{-1}{k+q}
$$

and

$\operatorname{Im} \int_{-\infty(1-i \epsilon)}^{0} d \eta^{\prime}\left(\frac{1}{\eta^{\prime 2}}-\frac{i(k+q)}{\eta^{\prime}}\right) e^{i(k+q) \eta^{\prime}}=-(k+q)$,

the integral over $\eta^{\prime}$ in Eq. (13) is calculated yielding

$$
\begin{aligned}
& \Delta\left\langle\delta \phi_{\mathbf{k}}\left(t_{e}\right) \delta \phi_{\mathbf{q}}\left(t_{e}\right)\right\rangle \\
& =\frac{\epsilon \pi H^{2}}{k^{3} q^{3}} \delta\left(k_{z}+q_{z}\right)\left(\frac{k q}{k+q}-(k+q)\right) \\
& \quad \times\left[\sum_{i \neq j} k_{j} q_{j} F_{i i}\left(\mathbf{k}_{\perp}+\mathbf{q}_{\perp}\right)-k_{i} q_{j} F_{i j}\left(\mathbf{k}_{\perp}+\mathbf{q}_{\perp}\right)\right],
\end{aligned}
$$

in which we have defined

$$
\begin{gathered}
F_{i j}\left(\mathbf{k}_{\perp}\right) \equiv \int d^{2} \mathbf{x} \exp \left(i \mathbf{k}_{\perp} \cdot \mathbf{x}_{\perp}\right) \frac{x_{i} x_{j}}{x^{2}+y^{2}}, \\
i, j \in\{1,2\} .
\end{gathered}
$$

With some effort, one can check that [40]

$F_{i j}\left(\mathbf{k}_{\perp}\right)=2 \pi^{2} \delta_{i j} \delta^{2}\left(\mathbf{k}_{\perp}\right)+\frac{4 \pi}{k_{1}^{2}+k_{2}^{2}}\left(\frac{\delta_{i j}}{2}-\frac{k_{i} k_{j}}{k_{1}^{2}+k_{2}^{2}}\right)$.

Plugging the above form of $F_{i j}\left(\mathbf{k}_{\perp}\right)$ in Eq. (18), and noting that the curvature perturbation $\mathcal{R}$ is related to $\delta \phi$ via $\mathcal{R}=-H \delta \phi / \dot{\phi}$, the curvature perturbations two-point correlation function in the presence of a cosmic string is obtained to be [40]

$$
\begin{aligned}
\left\langle\mathcal{R}_{\mathbf{k}}\left(t_{e}\right) \mathcal{R}_{\mathbf{q}}\left(t_{e}\right)\right\rangle= & \left(\frac{H^{2}}{\dot{\phi}}\right)^{2}\left\{\frac{(2 \pi)^{3}}{k^{3}} \delta^{3}(\mathbf{k}+\mathbf{q})\right. \\
& -\epsilon \pi\left(\frac{k^{2}+q^{2}+k q}{k^{3} q^{3}(k+q)}\right) \delta\left(k_{z}+q_{z}\right) \\
& \times\left[2 \pi^{2} \mathbf{k}_{\perp} \cdot \mathbf{q}_{\perp} \delta^{2}\left(\mathbf{k}_{\perp}+\mathbf{q}_{\perp}\right)\right. \\
& -\frac{4 \pi}{\left(\mathbf{k}_{\perp}+\mathbf{q}_{\perp}\right)^{2}}\left(\frac{\mathbf{k}_{\perp} \cdot \mathbf{q}_{\perp}}{2}\right. \\
& \left.\left.\left.-\frac{\mathbf{k}_{\perp} \cdot\left(\mathbf{k}_{\perp}+\mathbf{q}_{\perp}\right) \mathbf{q}_{\perp} \cdot\left(\mathbf{k}_{\perp}+\mathbf{q}_{\perp}\right)}{\left(\mathbf{k}_{\perp}+\mathbf{q}_{\perp}\right)^{2}}\right)\right]\right\} .
\end{aligned}
$$

For comparison, we have also added the leading isotropic and homogenous contribution from the inflaton field itself as given by the first term in Eq. (21), while the corrections from the string are given by the last three terms.

The structure of the symmetries of the two-point correlation function is somewhat nontrivial. The full $S O(3)$ rotation is broken to the subset of two-dimensional rotation in the $x y$ plane. One can easily see that all three terms from the corrections of string, the last line in Eq. (21), are invariant under rotation only around the string. As for the translation invariance, only the first term out of these three contributions retains the full threedimensional translation invariance because it has the three-dimensional Dirac delta function $\delta^{3}(\mathbf{k}+\mathbf{q})$. The last two corrections from the string break the translation invariance in the plane perpendicular to string as they have only $\delta\left(k_{z}+q_{z}\right)$. Since the string loses the full rotation and translation invariances, its corrections to the curvature perturbation power spectrum are a mixture of anisotropies and inhomogeneities. Therefore, the asymmetries generated by a cosmic string are more complicated than the simple dipole asymmetry modeled by Eq. (1) and cannot be captured just by the dipole amplitude $A_{d}$.

\section{QUADRUPOLE ANISOTROPY}

As we discussed above, the corrections to the power spectrum induced by cosmic strings have two distinct contributions. In particular, we see that the first term in the last line of Eq. (21) has the structure of a quadrupolar anisotropy as introduced in Eq. (2), in which the anisotropic (preferred) direction is the orientation of the cosmic string. As can be seen, the contribution of quadrupolar anisotropy is quite different than the contribution of the last two terms in Eq. (21), which mostly mimic a dipolar asymmetry. As we discussed before, the quadrupolar statistical anisotropy is associated with anisotropy at each point on the CMB map, while each CMB hemisphere has statistically the same power as the opposite hemisphere.

To calculate the amplitude of the quadrupolar anisotropy $g_{*}$, we compare the quadrupole term in the power spectrum with the isotropic power spectrum $P_{\mathcal{R}}^{(0)}$ given by the first term in Eq. (21), obtaining

$$
g_{*}=-\frac{3 \epsilon}{8} .
$$

The minus sign above is from the fact that $\sin ^{2} \theta=$ $1-\cos ^{2} \theta$.

This is an interesting prediction. We see that a cosmic string induces a quadrupole anisotropy in CMB maps that can be tested directly by cosmological observations. In particular, constraints from Planck observations [1,3,31] implies $\left|g_{*}\right| \lesssim 10^{-2}$, yielding $\epsilon \lesssim 10^{-2}$. Therefore, the scale of symmetry breaking responsible for the formation of 
strings during inflation cannot be much higher than the grand unified theory (GUT) scale. For example, if we assume that cosmic strings in the early Universe are in the form of a D or F string from string theory, then the mass scale of string theory cannot be much higher than the GUT scale. In addition, we see that the sign of $g_{*}$ is negative in our setup. Curiously, the sign of $g_{*}$ is also negative in all known models of anisotropic inflation [38].

\section{VARIANCE OF CURVATURE PERTURBATIONS}

Since the last two terms in Eq. (21) are not fully homogeneous, we expect them to induce an effective power asymmetry in CMB maps. The structure of these terms is too complicated to be used directly in an analytical study. A useful and practical tool is to look for the variance of curvature perturbations in real space $\left\langle\mathcal{R}(\mathbf{x})^{2}\right\rangle$. This provides insight about the magnitude and the form of power asymmetry generated by a cosmic string in temperature fluctuations. Following the analysis of Ref. [10], the Planck team has used the variance of temperature fluctuations [which is linearly related to $\mathcal{R}(\mathbf{x})$ ] as one of the measures of dipole asymmetry [5]. With this motivation in mind, we calculate $\left\langle\mathcal{R}(\mathbf{x})^{2}\right\rangle$ for our setup.

The correction in variance induced by the cosmic string is given by

$\Delta\left\langle\mathcal{R}(\mathbf{x})^{2}\right\rangle=\frac{1}{(2 \pi)^{6}} \iint \mathrm{d}^{3} \mathbf{k} \mathrm{d}^{3} \mathbf{q} e^{i(\mathbf{k}+\mathbf{q}) \cdot \mathbf{x}} \Delta\left\langle\mathcal{R}_{\mathbf{k}} \mathcal{R}_{\mathbf{q}}\right\rangle$.

The first correction from a string in Eq. (21) is fully translation invariant, so, as expected, it does not generate any position dependence in the variance; it only modifies the leading isotropic variance. Denoting its contribution to variance by $\Delta\left\langle\mathcal{R}(\mathbf{x})^{2}\right\rangle_{\text {hom }}$, we obtain

$$
\begin{aligned}
\Delta\left\langle\mathcal{R}(\mathbf{x})^{2}\right\rangle_{\mathrm{hom}} & =-\frac{3 \epsilon}{16}\left(\frac{H}{\dot{\phi}}\right)^{2} \int_{-\infty}^{\infty} d k_{z} \int_{0}^{\infty} d k_{\perp} \frac{k_{\perp}^{3}}{\left(k_{\perp}^{2}+k_{z}^{2}\right)^{5 / 2}} \\
& =-\epsilon \int d \ln k \mathcal{P}_{\mathcal{R}}{ }^{(0)}
\end{aligned}
$$

where $\mathcal{P}_{\mathcal{R}}{ }^{(0)}=\left(H^{2} / 2 \pi \dot{\phi}\right)^{2}$ is the isotropic power spectrum.

As expected, this has the same structure as the leading homogeneous variance. The constraint from the quadrupole anisotropy $\epsilon \ll 1$ guarantees that the corrections in isotropic and homogeneous variance induced from the first correction of the cosmic string are subleading compared to the contribution from an inflaton field. The interesting feature is that this contribution from strings has an opposite sign compared to the contribution from the inflaton. This may be good news to address the shortage of power on low $\ell$ as observed in Planck data. However, a careful data analysis must be performed to see whether the string can address the shortage of power on large scales while not changing the power on smaller scales and at the same time satisfying the constraints from the quadrupole statistical anisotropy.

The remaining two terms in Eq. (21) violate the translation invariance in the $x y$ plane and contribute nontrivially to the variance. Denoting these contributions by $\Delta\left\langle\mathcal{R}(\mathbf{x})^{2}\right\rangle_{\text {asym }}$, we have

$$
\begin{aligned}
\Delta\left\langle\mathcal{R}(\mathbf{x})^{2}\right\rangle_{\text {asym }}= & -\left.\epsilon\left(\frac{H^{2}}{\dot{\phi}}\right)^{2} \int \frac{d^{2} \mathbf{k}_{\perp} d^{2} \mathbf{q}_{\perp} d k_{z}}{(2 \pi)^{6}} e^{i\left(\mathbf{k}_{\perp}+\mathbf{q}_{\perp}\right) \cdot \mathbf{x}_{\perp}} \frac{4 \pi}{\left(\mathbf{k}_{\perp}+\mathbf{q}_{\perp}\right)^{2}}\left(\frac{k^{2}+q^{2}+k q}{k^{3} q^{3}(k+q)}\right)\right|_{q_{z}=-k_{z}} \\
& \times\left[\frac{1}{2} \mathbf{k}_{\perp} \cdot \mathbf{q}_{\perp}-\frac{1}{\left(\mathbf{k}_{\perp}+\mathbf{q}_{\perp}\right)^{2}} \mathbf{k}_{\perp} .\left(\mathbf{k}_{\perp}+\mathbf{q}_{\perp}\right) \mathbf{q}_{\perp} .\left(\mathbf{k}_{\perp}+\mathbf{q}_{\perp}\right)\right],
\end{aligned}
$$

in which the delta function $\delta\left(k_{z}+q_{z}\right)$ has been used to remove the integration over $q_{z}$. This also means that inside the integral we have $k=\sqrt{\mathbf{k}_{\perp}^{2}+k_{z}^{2}}$ and $q=\sqrt{\mathbf{q}_{\perp}^{2}+k_{z}^{2}}$.

Fortunately, the integral over $k_{z}$ can be taken analytically where

$$
\int_{-\infty}^{\infty} \frac{\mathbf{k}_{\perp}^{2}+\mathbf{q}_{\perp}^{2}+2 k_{z}^{2}+\left(\mathbf{k}_{\perp}^{2}+k_{z}^{2}\right)^{1 / 2}\left(\mathbf{q}_{\perp}^{2}+k_{z}^{2}\right)^{1 / 2}}{\left(\mathbf{k}_{\perp}^{2}+k_{z}^{2}\right)^{3 / 2}\left(\mathbf{q}_{\perp}^{2}+k_{z}^{2}\right)^{3 / 2}\left[\left(\mathbf{k}_{\perp}^{2}+k_{z}^{2}\right)^{1 / 2}+\left(\mathbf{q}_{\perp}^{2}+k_{z}^{2}\right)^{1 / 2}\right]} d k_{z}=\frac{2}{k_{\perp}^{2} q_{\perp}^{2}} .
$$

Plugging this in Eq. (25) yields

$$
\Delta\left\langle\mathcal{R}(\mathbf{x})^{2}\right\rangle_{\text {asym }}=-8 \pi \epsilon\left(\frac{H^{2}}{\dot{\phi}}\right)^{2} \int \frac{d^{2} \mathbf{k}_{\perp} d^{2} \mathbf{q}_{\perp}}{(2 \pi)^{6}} \frac{e^{i\left(\mathbf{k}_{\perp}+\mathbf{q}_{\perp}\right) \cdot \mathbf{x}_{\perp}}}{\left(\mathbf{k}_{\perp}+\mathbf{q}_{\perp}\right)^{2} k_{\perp}^{2} q_{\perp}^{2}}\left[\frac{1}{2} \mathbf{k}_{\perp} \cdot \mathbf{q}_{\perp}-\frac{1}{\left(\mathbf{k}_{\perp}+\mathbf{q}_{\perp}\right)^{2}} \mathbf{k}_{\perp} \cdot\left(\mathbf{k}_{\perp}+\mathbf{q}_{\perp}\right) \mathbf{q}_{\perp} .\left(\mathbf{k}_{\perp}+\mathbf{q}_{\perp}\right)\right] .
$$


The above expression for variance looks too complicated to be handled analytically. However, useful information can be obtained by looking at its asymptotic behaviors. It is easy to see that the integral above has no UV divergence as the integrand oscillates rapidly, yielding finite UV contributions. As for the IR behavior, we note that the integral is independent of scale in the sense that if we rescale all momenta and the measures by the factor $\left|\mathbf{x}_{\perp}\right|$ then the integral and the measure remain independent of scale while all scale dependence appears at the lower cutoff of the integral; i.e., it appears at the IR cutoff of the integral. Using this insight, we rescale all momenta by $\left|\mathbf{x}_{\perp}\right|$, writing the asymmetric variance as

$$
\begin{aligned}
\Delta\left\langle\mathcal{R}(\mathbf{x})^{2}\right\rangle_{\text {asym }}= & -8 \pi \epsilon\left(\frac{H^{2}}{\dot{\phi}}\right)^{2} \int_{\left|\mathbf{k}_{\perp}, \mathbf{q}_{\perp}\right|>\frac{\rho}{L}} \frac{d^{2} \mathbf{k}_{\perp} d^{2} \mathbf{q}_{\perp}}{(2 \pi)^{6} k_{\perp}^{2} q_{\perp}^{2}} \\
& \times e^{i\left(\mathbf{k}_{\perp}+\mathbf{q}_{\perp}\right) \cdot \hat{\mathbf{x}}_{\perp}} \frac{1}{\left(\mathbf{k}_{\perp}+\mathbf{q}_{\perp}\right)^{2}}\left[\frac{1}{2} \mathbf{k}_{\perp} \cdot \mathbf{q}_{\perp}\right. \\
& \left.-\frac{1}{\left(\mathbf{k}_{\perp}+\mathbf{q}_{\perp}\right)^{2}} \mathbf{k}_{\perp} .\left(\mathbf{k}_{\perp}+\mathbf{q}_{\perp}\right) \mathbf{q}_{\perp} \cdot\left(\mathbf{k}_{\perp}+\mathbf{q}_{\perp}\right)\right] .
\end{aligned}
$$

Here, $\rho \equiv x_{\perp}=\sqrt{x^{2}+y^{2}}$ is the perpendicular distance from the point $\mathbf{x}$ on the CMB sphere to the string, and $L$ represents the IR comoving cutoff of the setup, the size of an imaginary box that is bigger than the observable Universe.
With some effort, one can take the integral over $k_{2}$ and $q_{2}$ in Eq. (28), obtaining

$$
\begin{aligned}
\Delta\left\langle\mathcal{R}(\mathbf{x})^{2}\right\rangle_{\text {asym }}= & -\frac{\pi^{3} \epsilon}{(2 \pi)^{6}}\left(\frac{H^{2}}{\dot{\phi}}\right)^{2} \int d k_{1} d q_{1} \frac{e^{i\left(k_{1}+q_{1}\right) \rho}}{\left(k_{1}+q_{1}\right)^{2}} \\
& \times\left(\operatorname{sgn}\left(k_{1}+q_{1}\right)+\operatorname{sgn}\left(k_{1}\right)\right)\left(\operatorname{sgn}\left(q_{1}\right)\right. \\
& \left.+\operatorname{sgn}\left(2 k_{1}+q_{1}\right)\right) \\
= & \frac{-\pi^{3} \epsilon}{(2 \pi)^{6}}\left(\frac{H^{2}}{\dot{\phi}}\right)^{2} \int d k d q_{1} \frac{e^{i k \rho}}{k^{2}}(\operatorname{sgn}(k) \\
& \left.+\operatorname{sgn}\left(k-q_{1}\right)\right)\left(\operatorname{sgn}\left(q_{1}\right)+\operatorname{sgn}\left(2 k-q_{1}\right)\right) .
\end{aligned}
$$

Taking the integral and being careful on sign functions give the following result for the asymmetric variance:

$$
\begin{aligned}
\Delta\left\langle\mathcal{R}(\mathbf{x})^{2}\right\rangle_{\text {asym }}= & -\frac{\pi^{3} \epsilon}{(2 \pi)^{6}}\left(\frac{H^{2}}{\dot{\phi}}\right)^{2} \times 4\left(-2 \pi^{3}\right) \\
& \times \operatorname{Re}\left(\int_{\rho / L} \frac{d k}{k} \exp (i k)\right) \\
= & -\frac{\epsilon}{8 \pi^{3}}\left(\frac{H^{2}}{\dot{\phi}}\right)^{2} \ln (\rho / L) \\
= & -\frac{\epsilon}{2 \pi} \mathcal{P}_{\mathcal{R}}^{(0)} \ln (\rho / L) .
\end{aligned}
$$

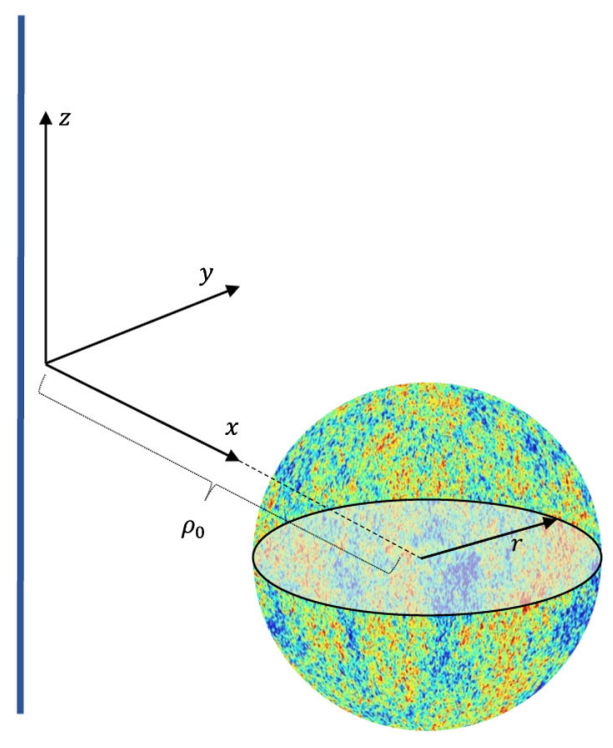

(a)

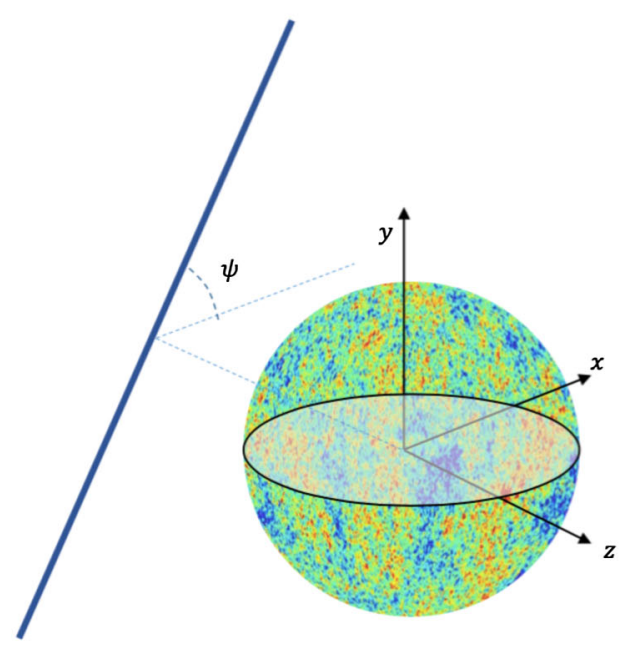

(b)

FIG. 1. (a) The original coordinate system in which the string is orientated along the $\hat{z}$ direction where we have calculated the corrections in the power spectrum. (b) The new coordinate in which we perform numerical analysis for variance. The direction of dipole asymmetry is toward the $-\hat{z}$ direction. In Galactic coordinates, we have $\hat{z}=(l, b)=\left(44^{\circ}, 22^{\circ}\right)$ and $\hat{x}=\left(44^{\circ},-68^{\circ}\right)$. 

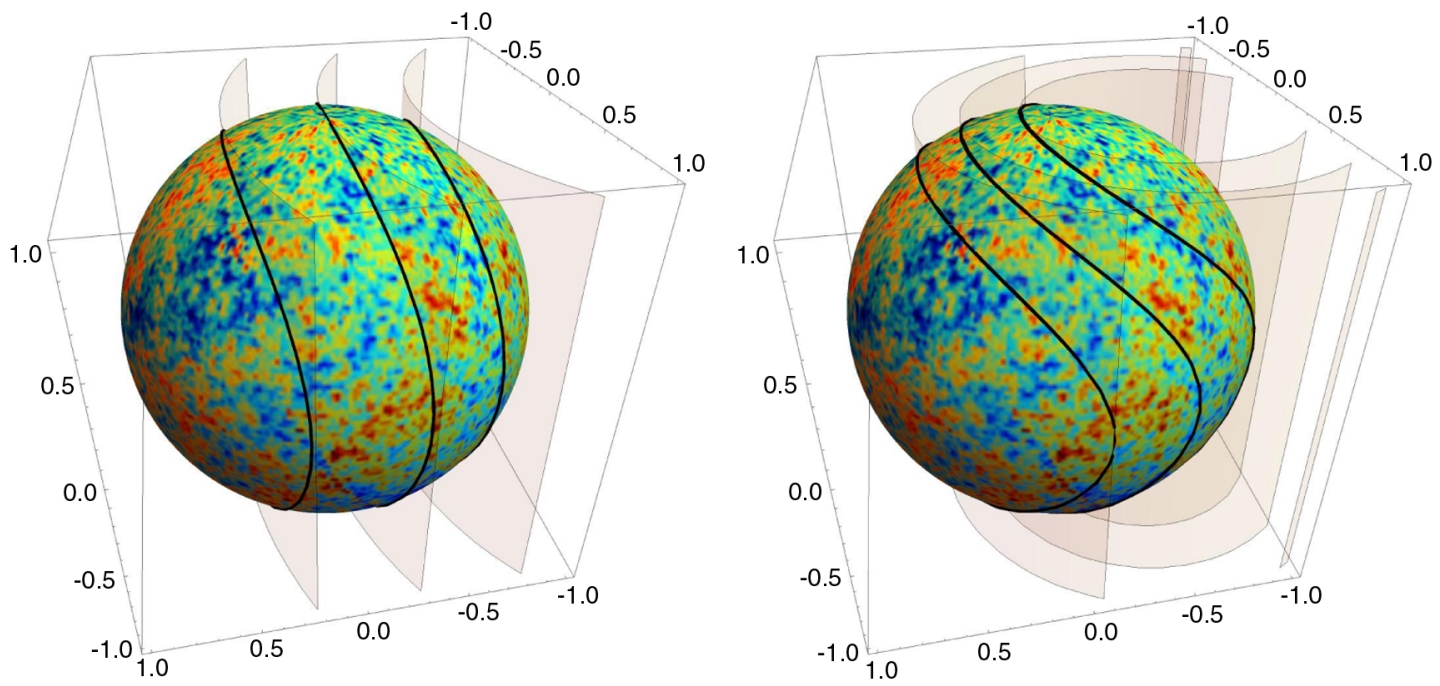

FIG. 2. The curves of equal variance on the CMB sphere induced by the cosmic string, left: $\kappa=0.5$, right: $\kappa=2$. These curves are obtained by the intersection of hypersurfaces $\rho=$ constant with the CMB sphere. The string is extended along the vertical direction, and the surfaces of constant $\rho$ are centered around the string.

\section{NUMERICAL RESULTS AND COMPARISON WITH OBSERVATIONS}

Having obtained the analytical estimate for the variance of curvature perturbation in Eq. (30), in this section, we look for the constraints on the model parameters by comparing our analytical result for the variance with the Planck data.

\section{A. Variance of the TT map}

In performing the variance analysis and comparing it with the data, it is better to change the coordinate to a convenient one for the CMB observer. So, instead of the configuration given in the left-hand side of Fig. 1, we use the coordinate presented in the right-hand side of that figure, in which the orientation of the string is measured by $\psi$, the angle with respect to a fixed $\hat{x}$ direction in the sky. In the new coordinate system, the anisotropic correction to the variance of the curvature perturbation from Eq. (30) (after removing the constant isotropic piece) is given by

$$
\begin{aligned}
\operatorname{Var}(\theta, \phi \mid \epsilon, \kappa, \psi)= & -\frac{\epsilon}{4 \pi} \ln \left(1+\kappa^{2} \sin ^{2} \theta \sin ^{2}(\phi-\psi)\right. \\
& \left.+\kappa^{2} \cos ^{2} \theta+2 \kappa \cos \theta\right)
\end{aligned}
$$

in which we have defined $\kappa \equiv r / \rho_{0}$, where $\rho_{0}$ is the distance between the string and the center of the $\mathrm{CMB}$ sphere and $r$ is the comoving radius of the CMB sphere as shown in Fig. 1. In Fig. 2, we have plotted the curves of constant variance on the CMB sphere. These curves are obtained by intersecting the hypersurfaces of constant $\rho$ with the CMB sphere. To compare (31) with the map of CMB variance, we compute the $a_{l m}$ multipoles associated with it:

$$
a_{l m} \equiv \int d \Omega Y_{l m}^{*}(\theta, \phi) \operatorname{Var}(\theta, \phi)
$$

In Ref. [10], the authors have constructed a map of variance out of the TT map of the Planck data. They have obtained the best-fit values for the direction of the variance asymmetry as well as the multipole moments of the variance map. In their analysis, they have assumed the $S O(2)$ symmetry for the map of the variance of temperature fluctuations. However, in our model, by locating an infinite cosmic string near our Hubble patch during inflation, we

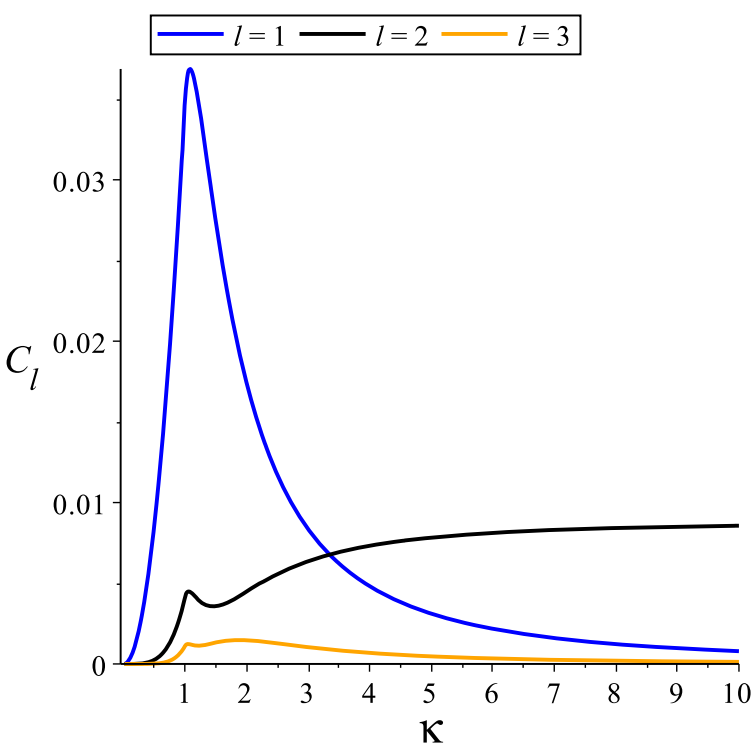

FIG. 3. Angular power spectrum of the variance as a function of $\kappa$ for dipole, quadrupole, and octupole with the normalization $\epsilon=1$. 

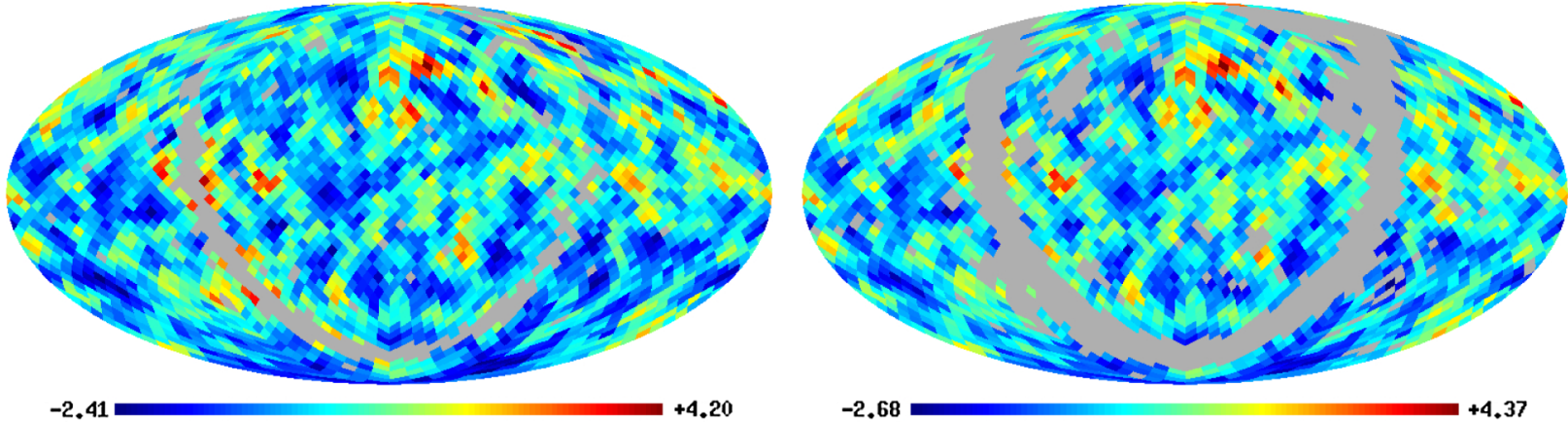

FIG. 4. Left: NILC masked variance map; right: SMICA masked variance map. They are masked by their individual masks and rotated such that the vertical direction lies in the direction of dipole variance asymmetry, namely, $(\ell, b)=(224,-22)$ in Galactic coordinates.

have spontaneously broken all rotational symmetries as well as the two-dimensional translational group on the plane perpendicular to the string.

Looking at Fig. 2, we realize that if $\kappa<1$, corresponding to the configuration in which the string lies outside of the CMB sphere, the contours of constant variance are not far from a set of parallel circles. On the other hand, we know that the map of $\mathrm{CMB}$ variance (like the one used in Ref. [10]) is very smooth as it has been averaged over few degrees circles. This suggests that, given a variance map, one can hardly distinguish between a string and a wall (an $\mathrm{SO}(2)$ symmetric configuration, like what is studied in
Ref. [27]), located somewhere outside of the CMB sphere. So, in what follows, to decrease the numerical cost of finding the best-fit parameters, we assume that in our model the direction $\hat{z}$ is the same as the direction of dipole asymmetry found in Ref. [10]. Later, we search for the bestfit value of $\psi$. Of course, by imposing this assumption, we implicitly exclude the possibility $\kappa \gg 1$ (configurations in which the string is close to the center of CMB). But this should not be a bad assumption because, roughly speaking, such configurations predict a nearly equal value of variance over the two opposite hemispheres, while the data prefer different values of variance on two opposite hemispheres.

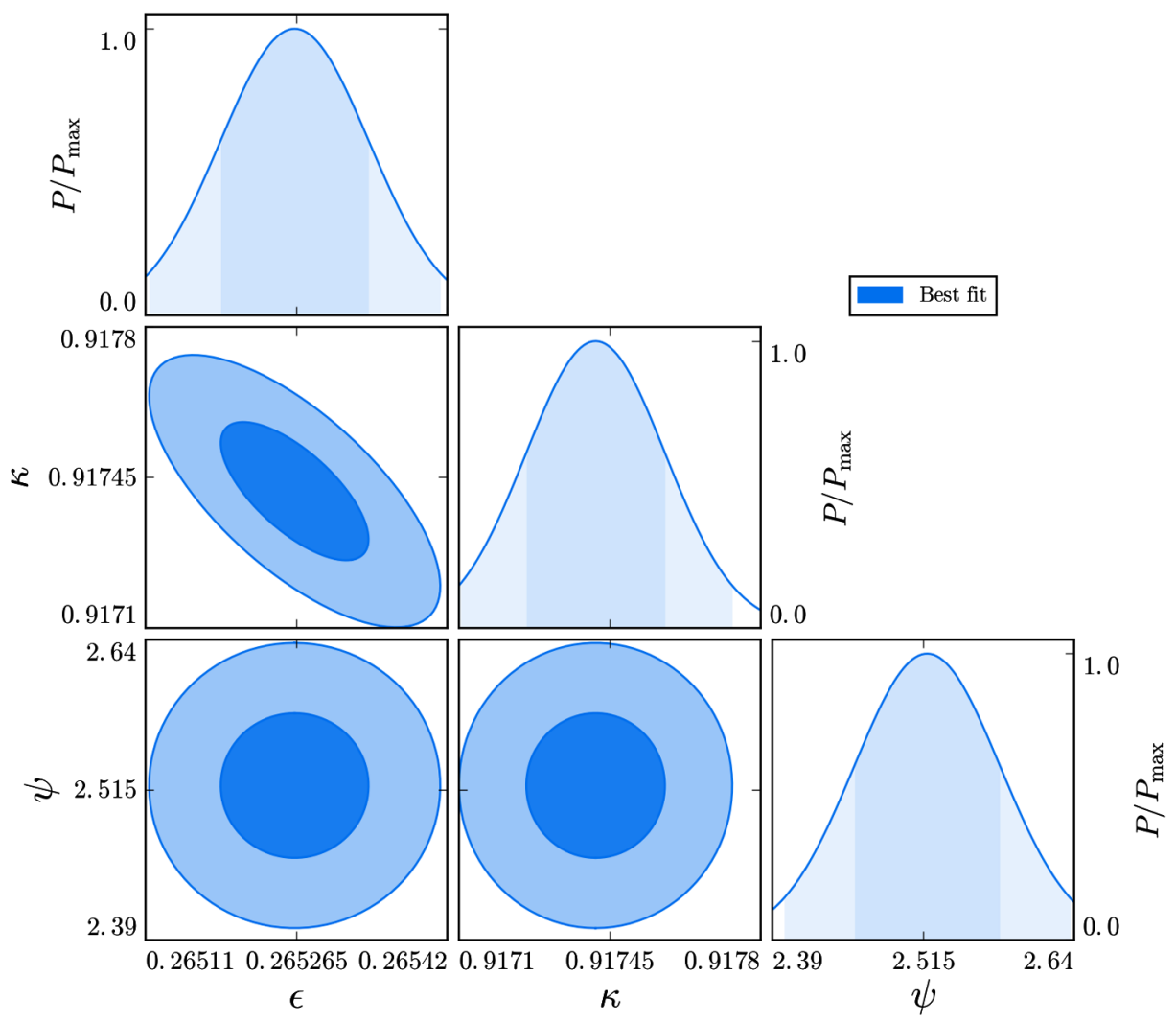

FIG. 5. The three-parameter Fisher analysis with the best values $(\epsilon, \kappa, \psi)=(0.265,0.917,2.518)$. 
Before going to search for the best fit of the direction of the string, namely, the angle $\psi$, we can calculate a tentative measure of variance asymmetry. As we explained above, for $\kappa<1$, the approximate $\mathrm{SO}(2)$ symmetry is recovered, so for these values of $\kappa$, we can average over $\psi$, or similarly we can sum over $m$. This would result in an averaged angular power spectrum,

$$
C_{l} \equiv \frac{1}{2 l+1} \sum_{m}\left|a_{l m}\right|^{2}
$$

This is the quantity computed in Ref. [10] for both simulations and the real data. In Fig. 3, we have plotted $C_{l}$ for the dipole, quadrupole, and octupole as a function of $\kappa$. We can immediately compare our predictions with the data reported in Ref. [10]. The data prefer a small $C_{2}$ and $C_{3}$, while allowing for $C_{1}$ as large as $\sim 0.01$. By considering only Fig. 3, there is no trouble in attaining such values for $C_{l}$. However, we must keep in mind not to rely on the values of $\kappa>1$ predicted here since, for such values of $\kappa, \psi$ cannot be averaged over.
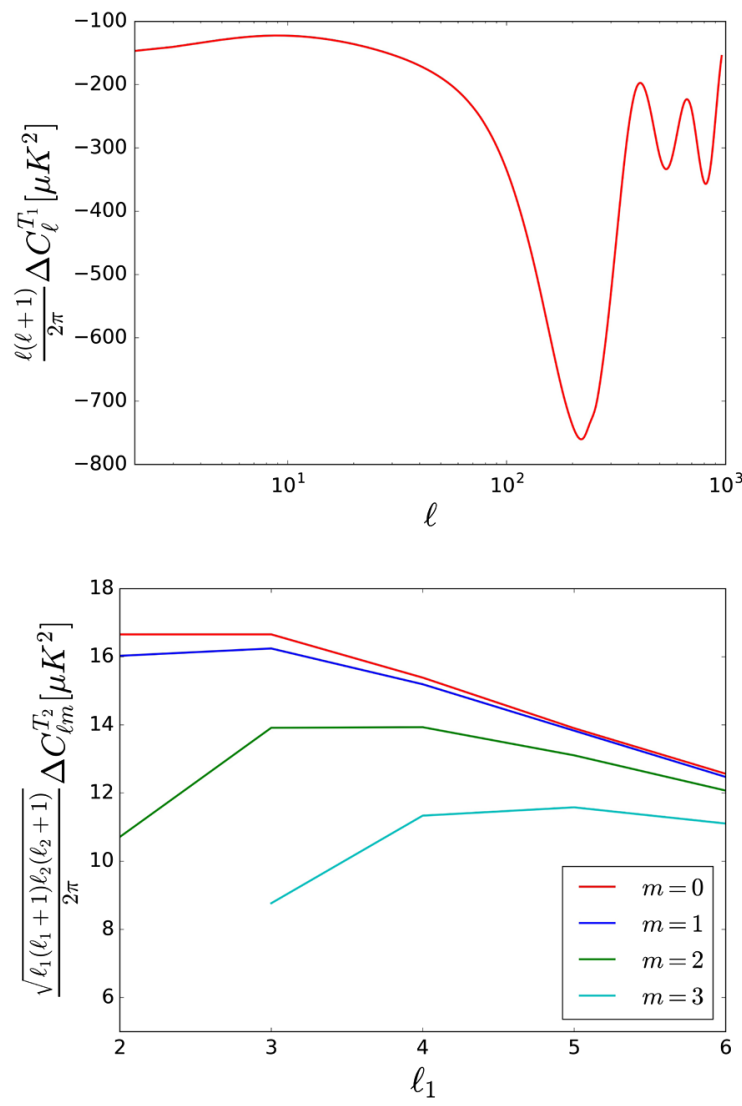

To perform a likelihood analysis and especially for taking the effects of $\psi$ into account, we have rotated the Planck map of the variance of fluctuations such that the direction of the reported dipole asymmetry (in spherical coordinates) is along the $-\hat{z}$ direction; see Figs. 1 and 4. Now, by means of a likelihood analysis and comparing $a_{l m}$ with the data, one can find the best-fit values for $\epsilon, \kappa$, and $\psi$. For this purpose, we used all CMB component separation algorithms, namely, Commander ruler, NILC, SMICA, and SEVEM maps of Planck DR-2 intensity maps and the corresponding masks for each component [60]. We extracted the variance map out of these maps by calculating the variance of the TT fluctuations over $6^{\circ}$ circles on the CMB sphere and applied the corresponding masks for each map. Afterward, we used those disks that had more than $90 \%$ unmasked pixels to construct a variance map and ignored other disks. Finally, we computed $a_{l m}$ for all of our variance maps and tried to maximize the likelihood function over the parametric space.

According to the comparison between simulation and data performed in Ref. [10], except for $\ell=1$, the higher
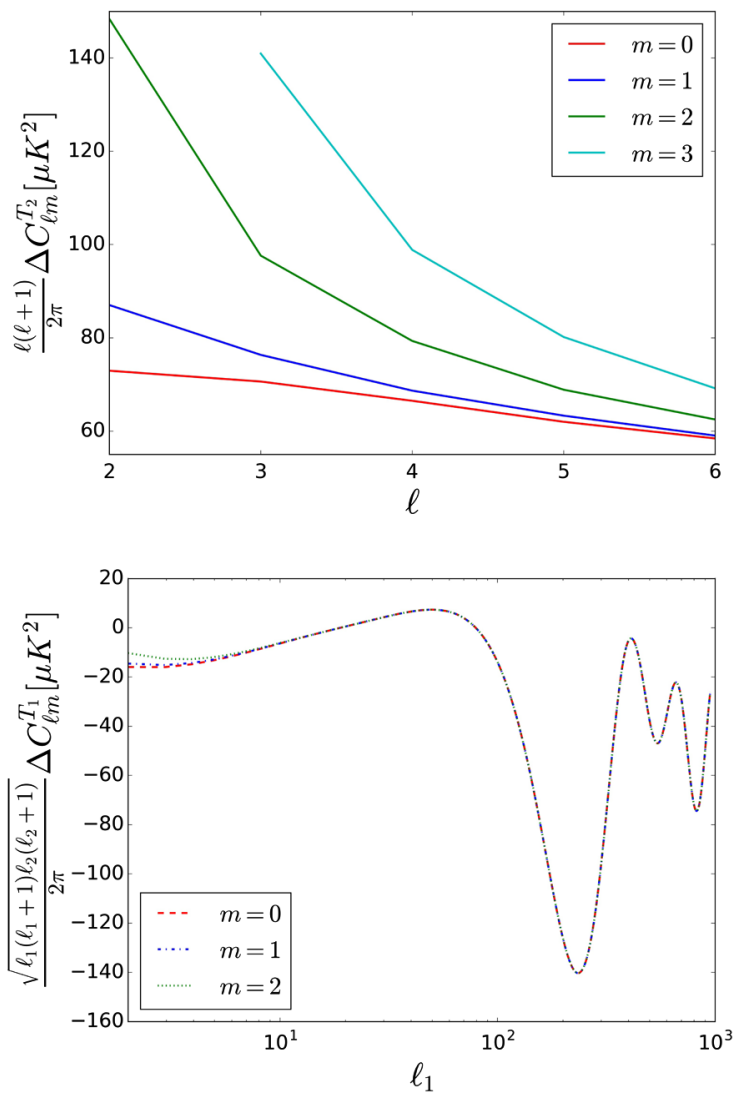

FIG. 6. Some elements of the first (homogenous) and second (inhomogeneous) parts of the power spectrum, Eq. (21), for the angular power spectrum matrix, $C_{(l, m)\left(l^{\prime}, m^{\prime}\right)}$, evaluated for the best-fit values found by variance analysis in Fig. V. Top left: the diagonal part of the first contribution with the sum over $m$. Top right: the diagonal part of the second contribution evaluated for different $m$. Since the computational cost of calculating this part is very high, we did not sum over all $\mathrm{m}$. Lower left: the $l_{2}=l_{1}+2$ elements of the second part of the angular power, evaluated for different $m$. Lower right: the $l_{2}=l_{1}+2$ element (the only nonzero off-diagonal element) of the first contribution for different $m$. 
multipoles of the anisotropy variance are consistent with zero and are not significant. Hence, in searching for the best-fit values of our model's parameters, we only look at $\ell<4$ multipoles. The best-fit values extracted out of this procedure are $(\epsilon, \kappa, \psi)=(0.265,0.917,2.518)$; see Fig. 5 . Taking higher $\ell$ s into account gives us a bad fit to the lower $\ell$ s, so we consider only $\ell<4$ multipoles.

There are two important points to mention. First, we see that this best-fit value, $\epsilon=0.265$, obtained from the dipolar asymmetry is an order of magnitude weaker than the constraint $\epsilon \lesssim 10^{-2}$ obtained from the quadrupolar anisotropy. Second, the best-fit value $\kappa=0.917$ corresponds to the configuration in which the string is very close to the CMB sphere. In a realistic situation, it requires fine-tuning, so one expects $\kappa$ to be somewhat different than unity.

\section{B. Angular spectrum of TT map}

Here, we perform the analysis of the CMB angular twopoint function.

Computing the angular two-point function of the TT map with the primordial curvature power spectrum, Eq. (21), is straightforward. The details of the formulas are reported in the Appendix. It is useful to decompose the primordial spectrum into two parts as represented in Eq. (A2). The first part does not violate the translational invariance and as mentioned before is simply a quadrupole term, while the second part breaks the translational invariance. These two parts have different contributions to the angular power spectrum; hence, in the following, we compute and plot each contribution separately.

The results shown in Fig. 6 are plotted for the best-fit values found in Fig. V, namely, $(\epsilon, \kappa)=(0.265,0.917)$. The second contribution, which violates translation invariance, decays rapidly for large $\ell \mathrm{s}$. As a result, the first contribution which is homogeneous dominates over the nonhomogeneous part for $\ell>3$ in diagonal elements. We also observe that the $\ell_{2}=\ell_{1}$ part of the second contribution is much bigger than its off-diagonal $l_{2}=l_{1}+2$ elements. Computing the second contribution is numerically too expensive, so we have calculated only its low multipole elements. ${ }^{1}$

\section{DISCUSSIONS}

In this work, we have looked for the imprints of a primordial cosmic string during inflation in generating

\footnotetext{
${ }^{1}$ We have to keep in mind that the theoretical value for the angular power spectrum depends on the coordinate we choose, due to the lack of rotational symmetry. Consequently, one cannot directly compare the diagonal $C_{l l^{\prime} m m^{\prime}}$ terms found here with the actual $C_{l}$ plots of Planck's data. Nevertheless, what we plot here should give a rough picture of what $C_{l}$ would look like if we were to rotate our TT map in order to match the coordinate in Planck's map and if we were to average properly over $m$ and $m^{\prime}$.
}

statistical anisotropy and power asymmetry. The question of looking for the effects of cosmic strings in the early Universe is very well motivated. Cosmic strings can be generated from a $U(1)$ symmetry breaking during inflation. Alternatively, they can be the F and D strings of superstring theory. Either way, constraining the tension of cosmic string directly constrains the mass scale of the corresponding underlying theories responsible for their formation.

The contribution of a cosmic string to the curvature power spectrum has two distinct parts. The first part is homogenous and has the form of a quadrupolar statistical anisotropy. Comparing with the Planck constraints on the amplitude of quadrupolar anisotropy, we obtain the upper bound $G \mu \lesssim 10^{-2}$ so the energy scale of the underlying theories generating cosmic string cannot be significantly higher than the GUT scale. The second contribution of a cosmic string to the curvature power spectrum breaks the translation invariance in the plane perpendicular to string. This contributes to asymmetry in the variance of curvature perturbations. The resulting constraint on the tension of cosmic string $G \mu \sim 10^{-1}$ is about an order of magnitude weaker than the constraint from the quadrupolar anisotropy.

By comparison, we comment that the bound on the tension of cosmic strings in a network formed in a symmetry breaking after inflation is much stronger, $G \mu \lesssim 10^{-7}$. This bound is obtained by assuming that the network of cosmic strings generates a fraction of temperature anisotropies in CMB maps, say less than $10 \%$ of the total power spectrum; see, for example, Refs. [53-56]. However, in our picture, the assumption is that the primordial string evaporates during reheating so it does not contribute actively to the temperature anisotropy in the subsequent evolution of the Universe. In addition, a network of cosmic strings formed after inflation generates a featureless plateau in the $\mathrm{CMB}$ angular power spectrum predominantly on high $\ell$. But, as we have seen, the primordial string in our picture generates power asymmetry and quadrupolar anisotropy on low- $\ell$ regions. Therefore, the contributions of a network of strings formed after inflation and the primordial strings during inflation on the CMB power spectrum are quite distinct. Therefore, our results can only constrain the scale of symmetry breaking responsible for the formation of strings during inflation.

We have calculated the contribution of the two abovementioned terms in the CMB angular power spectrum. Because isotropy and homogeneity are broken, we will have off-diagonal contributions in the angular power spectrum. The contribution of the inhomogeneous part rapidly falls off with $\ell$ for both diagonal and off-diagonal parts. A dedicated data analysis is required to investigate the full effects of strings on CMB temperature and polarization maps.

In our analysis, we have considered the simple picture of an infinite straight string in a Hubble patch. In a realistic situation, one may encounter a network of cosmic strings 
during inflation. So, an interesting question is what the imprints of a network of cosmic strings with a mix of loops and long strings on the inflationary power spectrum would be. During inflation, the strings are diluted quickly, so if one waits for few $e$-folds, then our picture of a long straight string is justified. However, during the short transient regime when the strings are being diluted, the imprints of a network of cosmic strings in the inflationary power spectrum would be much more complicated than our results. It may be an interesting question to look for the transient effects of a network of cosmic strings during the early stage of inflation and to see whether a network of cosmic strings can address the anomalies on CMB maps.

\section{ACKNOWLEDGMENTS}

We thank Yashar Akrami, Jaume Garriga, Martin Kunz, and Alessio Notari for useful discussions. H. F. would like to thank the University of Barcelona for hospitality where this work was at its final stage. The computations were performed at University of Geneva on the Baobab cluster.

\section{APPENDIX: ANGULAR POWER SPECTRUM}

The relation between the primordial curvature perturbations and angular fluctuations of the CMB is given by

$$
a_{l m}=4 \pi i^{l} \int \frac{d^{3} k}{(2 \pi)^{3}} \Delta_{l}(k) \mathcal{R}_{\mathbf{k}} Y_{l m}(\hat{k}),
$$

where $\mathcal{R}_{\mathbf{k}}$ is the curvature perturbations of a particular mode.

As discussed in the main text, the corrections from cosmic strings in the power spectrum, given in Eq. (21), have two distinct parts:

$$
\Delta\left\langle\mathcal{R}_{\mathbf{k}} \mathcal{R}_{\mathbf{q}}^{*}\right\rangle=\mathcal{F}_{1}(\mathbf{k}) \delta^{3}(\mathbf{k}-\mathbf{q})+\mathcal{F}_{2}\left(\mathbf{k}_{\perp}, \mathbf{q}_{\perp}\right) \delta\left(k_{z}-q_{z}\right) .
$$

The term $\mathcal{F}_{1}$ violates the isotropy but not the homogeneity, while $\mathcal{F}_{2}$ violates both isotropy and homogeneity. These functions are given by the following formulas:

$$
\begin{aligned}
\mathcal{F}_{1}(\mathbf{k})= & 12 \pi^{5} \epsilon \mathcal{P}_{\mathcal{R}}^{(0)} \frac{\sin ^{2} \theta}{k^{3}} \\
\mathcal{F}_{2}\left(\mathbf{k}_{\perp}, \mathbf{q}_{\perp}\right)= & -(2 \pi)^{4} \epsilon \mathcal{P}_{\mathcal{R}}^{(0)} \frac{\exp \left(i\left(k_{1}-q_{1}\right) \rho\right)}{k^{3} q^{3}} \\
& \times\left(\frac{k^{2}+q^{2}+k q}{k+q}\right) \frac{1}{\left(\mathbf{k}_{\perp}-\mathbf{q}_{\perp}\right)^{2}} \\
& \times\left\{\frac{1}{2} \mathbf{k}_{\perp} \cdot \mathbf{q}_{\perp}-\frac{1}{\left(\mathbf{k}_{\perp}-\mathbf{q}_{\perp}\right)^{2}}\right. \\
& \left.\times \mathbf{k}_{\perp} .\left(\mathbf{k}_{\perp}-\mathbf{q}_{\perp}\right) \mathbf{q}_{\perp} \cdot\left(\mathbf{k}_{\perp}-\mathbf{q}_{\perp}\right)\right\} .
\end{aligned}
$$

The matrix elements of the TT anisotropies are ${ }^{2}$

$$
\begin{aligned}
C_{(l, m)\left(l^{\prime} m^{\prime}\right)}^{T T}= & \left\langle a_{l m} a_{l^{\prime} m^{\prime}}^{*}\right\rangle \\
= & (4 \pi)^{2} i^{l-l^{\prime}} \int \frac{d^{3} k d^{3} q}{(2 \pi)^{6}} \Delta_{l}(k) \Delta_{l^{\prime}}(q) \\
& \times Y_{l m}(\hat{k}) Y_{l^{\prime} m^{\prime}}^{*}(\hat{q})\left\langle\mathcal{R}_{\mathbf{k}} \mathcal{R}_{\mathbf{q}}^{*}\right\rangle .
\end{aligned}
$$

We separate the matrix elements due to different terms in Eq. (A2). The first piece contributes as

$$
\begin{aligned}
C_{(l, m)\left(l^{\prime} m^{\prime}\right)}^{I}= & (4 \pi)^{2} i^{l-l^{\prime}} \int \frac{d^{3} k}{(2 \pi)^{6}} \Delta_{l}(k) \Delta_{l^{\prime}}(k) \mathcal{F}_{1}(\mathbf{k}) \\
& \times Y_{l m}(\hat{k}) Y_{l^{\prime} m^{\prime}}^{*}(\hat{k}) .
\end{aligned}
$$

Hereafter, the following convention for spherical harmonics functions is being used:

$Y_{l m}(\theta, \phi)=\sqrt{\frac{(2 l+1)}{4 \pi} \frac{(l-m) !}{(l+m) !}} P_{l}^{m}(\cos \theta) \exp (i m \phi)$

Correspondingly, Eq. (A5) simplifies to

$$
\begin{aligned}
C_{(l, m)\left(l^{\prime} m^{\prime}\right)}^{I}= & \delta_{m m^{\prime}} \sqrt{(2 l+1) \frac{(l-m) !}{(l+m) !}} \\
& \times \sqrt{\left(2 l^{\prime}+1\right) \frac{\left(l^{\prime}-m\right) !}{\left(l^{\prime}+m\right) !}}(4 \pi) i^{l-l^{\prime}} \\
& \times \int \frac{k^{2} d k \sin \theta d \theta}{(2 \pi)^{5}} \Delta_{l}(k) \Delta_{l^{\prime}}(k) \\
& \times \mathcal{F}_{1}(k, \theta) P_{l}^{m}(\cos \theta) P_{l^{\prime}}^{m *}(\cos \theta) .
\end{aligned}
$$

As for the second term, we have

$$
\begin{aligned}
C_{(l, m)\left(l^{\prime} m^{\prime}\right)}^{I I}= & (4 \pi)^{2} i^{l-l^{\prime}} \int \frac{k^{2} d k d \phi \sin \theta d \theta}{(2 \pi)^{6}} \\
& \times Q d Q d \phi^{\prime} \Delta_{l}(k) \Delta_{l^{\prime}}\left(\sqrt{Q^{2}+k^{2} \cos ^{2} \theta}\right) \\
& \times Y_{l m}(\cos \theta, \phi) Y_{l^{\prime} m^{\prime}}^{*}\left(\frac{k \cos \theta}{\sqrt{Q^{2}+k^{2} \cos ^{2} \theta}}, \phi^{\prime}\right) \\
& \times \mathcal{F}_{2}\left(\mathbf{k}_{\perp}, \mathbf{q}_{\perp}\right) .
\end{aligned}
$$

These expressions are used in our analysis to calculate the angular power spectrum in generating Fig. 6 .

\footnotetext{
${ }^{2}$ Note that $a_{l m}$ depends on the coordinates system we work with as shown in Fig. 1.
} 
[1] P. A. R. Ade et al. (Planck Collaboration), Astron. Astrophys. 594, A20 (2016).

[2] P. A. R. Ade et al. (Planck Collaboration), Astron. Astrophys. 594, A13 (2016).

[3] P. A. R. Ade et al. (Planck Collaboration), Astron. Astrophys. 571, A22 (2014).

[4] P. A. R. Ade et al. (Planck Collaboration), Astron. Astrophys. 571, A23 (2014).

[5] P. A. R. Ade et al. (Planck Collaboration), Astron. Astrophys. 594, A16 (2016).

[6] H. K. Eriksen, F. K. Hansen, A. J. Banday, K. M. Gorski, and P. B. Lilje, Astrophys. J. 605, 14 (2004); 609, 1198(E) (2004).

[7] H. K. Eriksen et al., Astrophys. J. 656, 641 (2007).

[8] H. K. Eriksen, A. J. Banday, K. M. Gorski, F. K. Hansen, and P. B. Lilje, Astrophys. J. 660, L81 (2007).

[9] C. Gordon, W. Hu, D. Huterer, and T. M. Crawford, Phys. Rev. D 72, 103002 (2005).

[10] Y. Akrami, Y. Fantaye, A. Shafieloo, H. K. Eriksen, F. K. Hansen, A. J. Banday, and K. M. Gorski, Astrophys. J. 784, L42 (2014).

[11] S. Aiola, B. Wang, A. Kosowsky, T. Kahniashvili, and H. Firouzjahi, Phys. Rev. D 92, 063008 (2015).

[12] S. Mukherjee, P. K. Aluri, S. Das, S. Shaikh, and T. Souradeep, J. Cosmol. Astropart. Phys. 06 (2016) 042.

[13] S. Mukherjee and T. Souradeep, Phys. Rev. Lett. 116, 221301 (2016).

[14] S. Adhikari, Mon. Not. R. Astron. Soc. 446, 4232 (2015).

[15] M. Quartin and A. Notari, J. Cosmol. Astropart. Phys. 01 (2015) 008; A. Notari, M. Quartin, and R. Catena, J. Cosmol. Astropart. Phys. 03 (2014) 019; O. Roldan, A. Notari, and M. Quartin, J. Cosmol. Astropart. Phys. 06 (2016) 026.

[16] M. Shiraishi, N. S. Sugiyama, and T. Okumura, Phys. Rev. D 95, 063508 (2017).

[17] M. Shiraishi, J. B. Muoz, M. Kamionkowski, and A. Raccanelli, Phys. Rev. D 93, 103506 (2016).

[18] S. Baghram, A. A. Abolhasani, H. Firouzjahi, and M. H. Namjoo, J. Cosmol. Astropart. Phys. 12 (2014) 036.

[19] F. Hassani, S. Baghram, and H. Firouzjahi, J. Cosmol. Astropart. Phys. 05 (2016) 044.

[20] J. P. Zibin and D. Contreras, Phys. Rev. D 95, 063011 (2017).

[21] S. Yasini and E. Pierpaoli, arXiv:1610.00015.

[22] A. L. Erickcek, M. Kamionkowski, and S. M. Carroll, Phys. Rev. D 78, 123520 (2008); A. L. Erickcek, S. M. Carroll, and M. Kamionkowski, Phys. Rev. D 78, 083012 (2008); A. L. Erickcek, C. M. Hirata, and M. Kamionkowski, Phys. Rev. D 80, 083507 (2009); L. Dai, D. Jeong, M. Kamionkowski, and J. Chluba, Phys. Rev. D 87, 123005 (2013).

[23] J. M. Maldacena, J. High Energy Phys. 05 (2003) 013.

[24] M. H. Namjoo, S. Baghram, and H. Firouzjahi, Phys. Rev. D 88, 083527 (2013); A. A. Abolhasani, S. Baghram, H. Firouzjahi, and M. H. Namjoo, Phys. Rev. D 89, 063511 (2014); M. H. Namjoo, A. A. Abolhasani, S. Baghram, and H. Firouzjahi, J. Cosmol. Astropart. Phys. 08 (2014) 002.

[25] C. T. Byrnes, D. Regan, D. Seery, and E. R. M. Tarrant, J. Cosmol. Astropart. Phys. 06 (2016) 025.

[26] D. H. Lyth, J. Cosmol. Astropart. Phys. 08 (2013) 007; J. F. Donoghue, K. Dutta, and A. Ross, Phys. Rev. D 80, 023526
(2009); L. Wang and A. Mazumdar, Phys. Rev. D 88, 023512 (2013); A. Mazumdar and L. Wang, J. Cosmol. Astropart. Phys. 10 (2013) 049; M. H. Namjoo, A. A. Abolhasani, H. Assadullahi, S. Baghram, H. Firouzjahi, and D. Wands, J. Cosmol. Astropart. Phys. 05 (2015) 015; H. Assadullahi, H. Firouzjahi, M. H. Namjoo, and D. Wands, J. Cosmol. Astropart. Phys. 04 (2015) 017; H. Firouzjahi, J. O. Gong, and M. H. Namjoo, J. Cosmol. Astropart. Phys. 11 (2014) 037; M. Zarei, Eur. Phys. J. C 75, 268 (2015); J. McDonald, J. Cosmol. Astropart. Phys. 07 (2013) 043; 11 (2013) 041; Phys. Rev. D 89, 127303 (2014); S. Kanno, M. Sasaki, and T. Tanaka, Prog. Theor. Exp. Phys. 2013, 111E01 (2013); A. R. Liddle and M. Cortes, Phys. Rev. Lett. 111, 111302 (2013); T. Kobayashi, M. Cores, and A. R. Liddle, J. Cosmol. Astropart. Phys. 05 (2015) 029; G. D’Amico, R. Gobbetti, M. Kleban, and M. Schillo, J. Cosmol. Astropart. Phys. 11 (2013) 013; Z.-G. Liu, Z.-K. Guo, and Y.-S. Piao, Phys. Rev. D 88, 063539 (2013); Eur. Phys. J. C 74, 3006 (2014); Y. F. Cai, W. Zhao, and Y. Zhang, Phys. Rev. D 89, 023005 (2014); Z. Chang, X. Li, and S. Wang, Chin. Phys. C 39, 055101 (2015); K. Kohri, C. M. Lin, and T. Matsuda, J. Cosmol. Astropart. Phys. 08 (2014) 026; Z. Chang and S. Wang, arXiv:1312.6575; Z. Kenton, D. J. Mulryne, and S. Thomas, Phys. Rev. D 92, 023505 (2015); S. Mukherjee, Phys. Rev. D 91, 062002 (2015); A. Ashoorioon and T. Koivisto, Phys. Rev. D 94, 043009 (2016); S. Adhikari, S. Shandera, and A. L. Erickcek, Phys. Rev. D 93, 023524 (2016); C. T. Byrnes and E. R. M. Tarrant, J. Cosmol. Astropart. Phys. 07 (2015) 007; C. T. Byrnes, D. Regan, D. Seery, and E. R. M. Tarrant, Phys. Rev. D 93, 123003 (2016); C. Byrnes, G. Doménech, M. Sasaki, and T. Takahashi, J. Cosmol. Astropart. Phys. 12 (2016) 020.

[27] S. Jazayeri, Y. Akrami, H. Firouzjahi, A. R. Solomon, and Y. Wang, J. Cosmol. Astropart. Phys. 11 (2014) 044.

[28] H. Firouzjahi, A. Karami, and T. Rostami, J. Cosmol. Astropart. Phys. 10 (2016) 023.

[29] L. Ackerman, S. M. Carroll, and M. B. Wise, Phys. Rev. D 75, 083502 (2007); 80, 069901(E) (2009).

[30] A. R. Pullen and M. Kamionkowski, Phys. Rev. D 76, 103529 (2007).

[31] J. Kim and E. Komatsu, Phys. Rev. D 88, 101301 (2013).

[32] M.-a. Watanabe, S. Kanno, and J. Soda, Phys. Rev. Lett. 102, 191302 (2009).

[33] M.-a. Watanabe, S. Kanno, and J. Soda, Prog. Theor. Phys. 123, 1041 (2010).

[34] N. Bartolo, S. Matarrese, M. Peloso, and A. Ricciardone, Phys. Rev. D 87, 023504 (2013).

[35] M. Shiraishi, E. Komatsu, M. Peloso, and N. Barnaby, J. Cosmol. Astropart. Phys. 05 (2013) 002.

[36] R. Emami, arXiv:1511.01683.

[37] A. A. Abolhasani, R. Emami, J. T. Firouzjaee, and H. Firouzjahi, J. Cosmol. Astropart. Phys. 08 (2013) 016.

[38] T. Rostami, A. Karami, and H. Firouzjahi, arXiv: 1702.03744.

[39] S. M. Carroll, C. Y. Tseng, and M. B. Wise, Phys. Rev. D 81, 083501 (2010).

[40] C. Y. Tseng and M. B. Wise, Phys. Rev. D 80, 103512 (2009).

[41] T. Prokopec and P. Reska, J. Cosmol. Astropart. Phys. 03 (2011) 050. 
[42] C. H. Wang, Y. H. Wu, and S. D. H. Hsu, Phys. Lett. B 713, 6 (2012).

[43] H. T. Cho, K. W. Ng, and I. C. Wang, Classical Quantum Gravity 28, 055004 (2011).

[44] H. T. Cho, K. W. Ng, and I. C. Wang, J. Cosmol. Astropart. Phys. 11 (2014) 023.

[45] T. W. B. Kibble, J. Phys. A 9, 1387 (1976).

[46] S. Sarangi and S.H.H. Tye, Phys. Lett. B 536, 185 (2002).

[47] N. T. Jones, H. Stoica, and S. H. H. Tye, Phys. Lett. B 563, 6 (2003).

[48] E. J. Copeland, R. C. Myers, and J. Polchinski, J. High Energy Phys. 06 (2004) 013.

[49] H. Firouzjahi and S.-H. H. Tye, J. Cosmol. Astropart. Phys. 03 (2005) 009.

[50] H. Firouzjahi, L. Leblond, and S.-H. Henry Tye, J. High Energy Phys. 05 (2006) 047.
[51] D. F. Chernoff and S.-H. H. Tye, Int. J. Mod. Phys. D 24, 1530010 (2015).

[52] S.-H. H. Tye, Lect. Notes Phys. 737, 949 (2008).

[53] J. Lizarraga, J. Urrestilla, D. Daverio, M. Hindmarsh, and M. Kunz, J. Cosmol. Astropart. Phys. 10 (2016) 042.

[54] T. Charnock, A. Avgoustidis, E. J. Copeland, and A. Moss, Phys. Rev. D 93, 123503 (2016).

[55] J. Lizarraga, J. Urrestilla, D. Daverio, M. Hindmarsh, M. Kunz, and A. R. Liddle, Phys. Rev. D 90, 103504 (2014).

[56] A. Lazanu and P. Shellard, J. Cosmol. Astropart. Phys. 02 (2015) 024.

[57] A. Vilenkin and E. P. S. Shellard, Cosmic Strings and Other Topological Defects (Cambridge University Press, 2000).

[58] S. Weinberg, Phys. Rev. D 72, 043514 (2005).

[59] R. Gregory, Phys. Rev. D 39, 2108 (1989).

[60] R. Adam et al. (Planck Collaboration), Astron. Astrophys. 594, A9 (2016). 